American academic institutions. The past several decades have seen the classification shaped to meet the policy objectives of the sponsors - the Carnegie Foundation for the Advancement of Teaching. The new sponsor, the Lumina Foundation, will no doubt shape the classification to suit its needs and advance its agenda — and the result is unlikely to be relevant to the original purpose of the classification.

\section{What Is Really Needed}

It is surprising that, in the four decades since Clark Kerr conceptualized the Carnegie Classification, no one has stepped forward to provide a clear and reasonably objective and comprehensive guide to the more than 4,500 postsecondary institutions in the United States. Resurrecting the basic purpose and organization of Kerr's original Carnegie Classification is not rocket science, nor would it be extraordinarily expensive.

It is of course true that the postsecondary education has become more complex. How would one deal with the for-profit sector? - probably by adding a special category for them. Many community colleges now offer four-year bachelor's degrees, but their basic purpose and organization has not essentially changed. There are a larger number of specialized schools, and many colleges and universities have expanded and diversified their degree and other offerings. Technology has to some extent become part of teaching programs of some postsecondary institutions - and the massive open online course (MOOC) revolution continues to unfold. Research productivity has grown dramatically, and research is reported in more ways. Intellectual property of all kinds has become more central to the academic enterprise-at least in the research university sector.

Yet, the basic elements of the original classificationthose that help to determine the main purposes and functions of postsecondary institutions-remain largely unchanged, if somewhat more complicated to describe. The key metrics are clear enough:

-Student enrollment

-Degrees awarded

-Types of degrees offered

-Number of faculty, full-time and part-time

-Income from research and intellectual property

- Research productivity

-Internationalization as measured by student mobility. A few more might be added-but again, simplicity is the watchword.

The types of institutions- 6 main and 8 major subcategories-seem about right. These might be expanded somewhat to accommodate the growth in complexity and diversity of the system. Later iterations confusingly expanded the categories, in part to reflect the policy and philosophical orientations of the foundation. The basic purpose of the classification will be best served by keeping the institutional typology as simple and straightforward as possible.

While it is clear that these metrics may not provide a sophisticated or complete measure of each institution-and they require additional definitions - they will provide basic information that will make reasonably categorization possible. They lack the philosophical and policy orientations that have crept into the Carnegie Classification in recent years, and return the enterprise to its original purpose-describing the richness, diversity, and complexity of the American higher education landscape.

\section{MOOCs in the Developing World: Hope or Hype?}

\section{Ben WiLdavsky}

Ben Wildavsky is director of higher education studies at the Nelson A. Rockefeller Institute of Government, State University of New York, and policy professor at the State University of New York-Albany. This essay is adapted from an article in the May/June 2014 issue of International Educator. E-mail: ben.wildavsky@suny.edu.

$\mathrm{T}$ he first university class to carry the unwieldy acronym of the massive open online course (MOOC) was created in 2008 at the University of Manitoba. But the muchtouted MOOC revolution did not truly take off until several years later, with the emergence of the Big Three: for-profits Udacity and Coursera-educational organizations, and the nonprofit Harvard-Massachusetts Institute of Technology collaboration EdX-an online course. They remain the best-known players today, typically featuring free noncredit classes that offer some mixture of short video segments, quizzes, online discussion boards, and writing assignments graded by peers.

From the start, the global potential of MOOCs, particularly in the developing world, was a large part of what made them so captivating. When two renowned computer scientists at Stanford University took their Introduction to Artificial Intelligence class online and offered it free to students anywhere in the world, they quickly attracted $\mathrm{i} 60,000 \mathrm{stu}-$ dents from igo countries. There were famously more students from Lithuania enrolled in the class than there are members of Stanford's entire student body.

Since then, other MOOCs have expanded on a massive scale. Coursera, the largest MOOC provider, has registered Io million students in courses offered by more than Ioo universities. Its business model remains unproven, but it 
is a sufficiently attractive prospect to have received $\$ 85$ million in venture funding. Along with growth has come evergreater ambition. Coursera proclaims a vision of the future in which "everyone has access to a world-class education that has so far been available to a select few."

\section{The Skeptics}

However, if the advent of MOOCs was accompanied by enormous enthusiasm about their potential to democratize access to high-quality education in poor countries, it was not long before MOOC hype gave way to MOOC hate, or at least intense skepticism. Critics argue that MOOC boosters have made vastly overblown claims about who really benefits from free, large-scale online classes. Moreover, they see MOOCs as poorly tailored to non-Western cultures and even as instruments of neocolonialism.

\section{From the start, the global potential of MOOCs, particularly in the developing world, was a large part of what made them so captivating.}

Are MOOCs really a boon to the developing world, or have they been oversold? The critics cite much evidence to bolster their cause. For one thing, most MOOC students already have degrees and live in developed countries. When the University of Pennsylvania surveyed the more than 400,000 active users of its Coursera classes, it found that two-thirds came from the United States and other Organization for Economic Cooperation and Development nations. These 34 industrialized countries account for a modest I8 percent of the world's population.

However, MOOCs do not appear to be reaching students with little postsecondary education. The same survey found that 83 percent of students taking Penn's Coursera classes already have two- or four-year degrees (and that about two-thirds of those in developing countries are male).

Moreover, MOOCs have notoriously high dropout rates. Just 5 percent of those enrolled in I7 EdX classes in 2012 and 2013 earned certificates of completion.

Last, detractors maintain the democratization of education promised by MOOC boosters falls short because it is based on the flawed assumption that the rest of the world will benefit from what MOOCs are selling. Critics call MOOCs elitist instruments of Western academic dominance that are not appropriately tailored to non-Western cultures and risk undermining local institutions and academic traditions.

\section{More Good Than Harm}

It is surely no surprise that the MOOC craze that peaked in 2012 has given way to so much skepticism. Some of the warnings critics offer deserve serious scrutiny. But MOOCs will likely do more good than harm in the developing world, particularly if they are not viewed as static but as evolving forms of technology-enabled pedagogy.

MOOC myth-busters are correct to note that non-Westerners with little education from low-income countries make up a distinct minority of MOOC students, and that completion rates are low. But these observations can themselves be misleading. MOOC enrollments are so large that even, say, a 90 percent noncompletion rate can still result in an eye-catching I0,000-plus students with certificates of completion. Also, many students counted as "dropouts" may have sampled course offerings without ever intending to complete.

Students' educational backgrounds, too, are not as universally privileged as first appearances might suggest. While two-thirds of EdX course registrants in 2012 and 2013 reported having post-high school education, that still leaves 223,000 with a high school education or less.

Moreover, it should be no surprise that wealthier, bettereducated people have dominated the first waves of MOOC enrollment. After all, the personal computer and Internet revolutions started with elites before gradually transforming broad swaths of society.

What about of alleged Western neocolonialism in MOOCs' academic content, institutional affiliation, and pedagogy? Perhaps the first response to such ideologically freighted criticism is that no one is being forced to sign up for MOOCs. Just as Western universities are enormous magnets for students from developing countries who have the means and motivation to attend them in person, online courses from the likes of Stanford University and Massachusetts Institute of Technology hold significant appeal.

\section{WORKS IN PROGRESS}

It is fine, to ask whether MOOCs can be effective pedagogically in a range of cultural contexts. Yet, the most useful way to think about MOOCs in the developing world is to view them as works in progress. In short, we are in a period of experimentation on a massive scale.

As in the United States, some MOOCs could end up leading to short-term, practical certificates rather than fullblown degrees. Some will end up appealing to learners who are primarily "browsers," akin to library users. For more engaged students, there is growing attention to blended models that make the best use of high-quality course con- 
tent, while giving students face-to-face instruction tailored to their own strengths and weaknesses.

In Africa, for example, where 93 percent of the collegeaged population is not in college, a range of MOOCs and MOOC-like ventures is serving students with blendedlearning classes. Finding the most appropriate technology is a challenge. Broadband Internet connections are often hard to access, making mobile phones the best way to reach some students. Development expert Guy Pfefferman notes that 25 million Africans had mobile phones in 200I-a number that jumped to 280 million by 2013 . In countries such as Ghana, Cameroon, Nigeria, and Tanzania, 80 percent or more of the population now owns mobile phones.

Against this backdrop, EdX has announced a partnership with Facebook to create a project called SocialEDU. The idea of the pilot program, which will start in Rwanda, is to go beyond today's MOOC technology to build a platform that capitalizes on readily available and inexpensive mobile devices. Content, provided by EdX, will be free. Facebook will handle the app and create the kind of mobile learning environment that many believe will be crucial to taking free, high-quality course offerings to scale in the developing world.

The combination of expanding educational aspirations, greatly improved technology, and more creative pedagogy will inevitably lead to more global experimentation with MOOCs, naysayers notwithstanding. MOOCs will surely need to evolve to serve students more effectively. But, the standard for new forms of higher education should not be whether they are perfect. It should be how they compare to the highly imperfect alternatives faced by many students, particularly in the world's poorest countries.

\section{Improving Engineering Edu- cation in Sub-Saharan Africa}

\section{Goolam Mohamedbhal}

Goolam Mohamedbhai is former Secretary General of the Association of African Universities. E-mail: g_t_mobhai@yahoo.co.uk. This article draws from a report commissioned by the World Bank: "Improving the Quality of Engineering Education in Sub-Saharan Africa," by Goolam Mohamedbhai. A longer version will appear in the International Journal of African Higher Education.

Sub-Saharan Africa has been experiencing robust eco$S$ nomic growth in recent years, attracting significant foreign investment. However, the foreign investment projects are handicapped by an acute shortage of domestic skilled labor, making it necessary to import foreign skills. For Africa to sustain its unprecedented economic growth and become competitive, the development of its human capital is paramount, especially in the areas of engineering and technology.

There is, in particular, an urgent need of engineering capacity in Africa for several reasons: For its infrastructural development to accompany its growth trajectory; for accelerating its industrial development, especially in manufacturing, so that it becomes a net exporter rather than importer of manufactured goods; for producing its ever-increasing needs in terms of energy to overcome the acute power shortages it experiences regularly; for empowering it to take control of the mining of its rich natural resources; and finally, for achieving the Millennium Development Goals.

\section{Current State of Engineering Education}

Several reports have been recently published on the provision of engineering education and training in African countries. In 20I2, the Royal Academy of Engineering published a comprehensive report on identifying engineering capacity needs in sub-Saharan Africa, based on an electronic survey of II3 professional engineers and 29 decision makers from I8 African countries, as well as interviews with I5 engineering project leaders in various African countries. In 2005, the African Technology Policy Studies Network published a report on the capacity of engineering education in Nigeria, Ghana, and Zimbabwe-using data obtained from questionnaires and interviews with a wide range of stakeholders. Also, in 20IO, UNESCO published a landmark report on engineering, with contributions from 120 experts around the world and with a special emphasis on the role of engineering in international development.

These studies reveal two key findings. First, there is a severe lack of engineering capacity in Africa, which has to rely heavily on imported expertise. This lack of capacity results from several sources: insufficient output from the training institutions to meet the countries' requirements; poor quality and lack of practical experience and skills of the graduates produced, which often make them unemployable; local presence of foreign engineering firms who prefer to import their own skilled labor; and the reluctance of the graduates to take up poorly paid positions in rural areas.

Second, there is an acute shortage of engineering technicians. Generally, for the effective operation of the engineering industry, the ratio professional engineers: technicians should be of the order of $1: 5$ or $1: 6$, indicating the need for a far greater number of technicians than engineers. In Africa, however, this ratio is more of the order of I:I or I:I.5. This could imply that a number of qualified engineers are underemployed and are working as techni- 